\title{
多电子原子或离子体系模型势理论中 旋-轨耦合系数的计算
}

\author{
王麓雅一文根䚾 \\ (湖南师范大学物理系, 长沙 410081)
}

\section{关键词量子化学、原子结构、势模型、旋一轨粴合}

应用郑能武所建议的近似描述多电子原子及离子的模型势理论 ${ }^{[1,2]}$, 作者曾导出了一个计 算任意幂次算符 $r^{k}$ 矩阵元的公式 ${ }^{[3]}$, 使用该式可以对各类跃迁振子强度进行计算. 进而,为了 能方便的计算多电子原子及离子任意激发态的旋-轨耦合能,本文利用正负幂平均值之间的关 系和各幂次平均值之间的关系, 导出了一个计算旋-轨棵合系数的简易公式.

\section{一、正负赛平均值之间的关系}

采用原子单位制,文献 $[1,2]$ 中的径向波函数可表示为

$$
R_{n l}(r)=N_{n l}\left(\begin{array}{c}
2 z^{*} r \\
n^{*}
\end{array}\right)^{l *} \exp \left(-\frac{z^{*}}{n^{*}} r\right) L_{n-i-1}^{2 i^{*}+1}\left(\frac{2 z^{*}}{n^{*}} r\right),
$$

些中

$$
\left\{\begin{array}{l}
z^{*}=\sqrt{(z-\sigma)^{2}+g \Delta z} \\
l^{*}=l+d, n^{*}=n+d
\end{array}\right.
$$

为修正的聂子数, $N_{n l}$ 为归一化常数.

文䰜[3]使用波函数 (1)式导出了平均值的计算式为

$$
\begin{aligned}
\left\langle n l\left|r^{k}\right| n l\right\rangle= & \left(\frac{n^{*}}{2 z^{*}}\right)^{k} \cdot \frac{(n-l-1) !}{2 n^{*}} \cdot \frac{\Gamma\left(2 l^{*}+k+3\right)}{\Gamma\left(n^{*}+l^{*}+1\right)} \\
& \cdot \sum_{m=1}^{n-l-1}\left(\begin{array}{c}
k+1 \\
n-l-1-m
\end{array}\right)^{2}\left(\begin{array}{c}
2 l^{*}+k+2+m \\
m
\end{array}\right),
\end{aligned}
$$

其中求和限为二项式系数的非零范围决定, 可得 $s^{\prime}=\max \{0, n-l-1-k-1\}$.

当 $k<-1$ 时,基于二项式系数约性质,(3)式应改为

$$
\begin{aligned}
\left\langle n l\left|r^{k}\right| n l\right\rangle= & \left(\frac{n^{*}}{2 z^{*}}\right)^{k} \cdot \frac{(n-l-1) !}{2 n^{*}} \cdot \frac{\Gamma\left(2 l^{*}+k+3\right)}{\Gamma\left(n^{*}+l^{*}+1\right)} \\
& \cdot \sum_{m=0}^{n-l-1}\left(\begin{array}{c}
-k+n-l-m-3 \\
n-l-1-m
\end{array}\right)^{2}\left(\begin{array}{c}
2 l^{*}+k+2+m \\
m
\end{array}\right) .
\end{aligned}
$$

进一步考虑(3)式与(4)式之间的关系, 我们令 $k+1=T \geqslant 0$ 代入 (3)式, 并令一 $(k+$ $2)=T \geqslant 0$ 代入(4)式, 经过适当变换后, 消去求和项, 可得如下正负幂平均值之间的关系:

$$
\left\langle n l\left|r^{T-1}\right| n l\right\rangle=\left(\frac{n^{*}}{2 z^{*}}\right)^{2 T+1} \cdot \frac{\Gamma\left(2 l^{*}+T+2\right)}{\Gamma\left(2 l^{*}-T+1\right)} \cdot\left\langle n l\left|r^{-r-2}\right| n l\right\rangle,
$$

由 $\Gamma$-函数的性质, $l^{*}>\frac{1}{2}(T-1)$. (5)式包含了类氢离子情形, 只要令式中 $n^{*}=n, l^{*}=$

本文 1991 年 5 月 17 日收到. 
$l, z^{*}=z$, 将可化为与 Bockasten ${ }^{[4]}$ 相闰的关系式.

\section{二、旋 轨耦合系数的计算}

仍采用原子单位制, 文献 [1]中的势函数可表示为

$$
V(r)=-\frac{z^{*}}{r}+\frac{B}{r^{2}},
$$

式中

$$
B=\frac{1}{2}[d(d+1)+2 d l] .
$$

旋-轨耦合系数篮要计算下式:

$$
\xi_{a l}=\langle n l|\xi(r)| n l\rangle,
$$

其中

$$
\xi(r)=\frac{1}{2 c^{2} r} \cdot \frac{d V(r)}{d r} .
$$

将势函数(6)式代入(9)式后,(8)式可得

$$
\xi_{n l}=\frac{1}{2 c^{2}}\left(z^{*}\left\langle n l\left|r^{-3}\right| n l\right\rangle-2 B\left\langle n l\left|r^{-4}\right| n l\right\rangle\right) .
$$

为了化简上式, 我们先求各幂次平均值之间的关系,利用 Hyper-Virial 定理

$$
\langle n l|[\hat{H}, \hat{W}]| n l\rangle=0,
$$

式叶 $\hat{H}$ 为 Hamilton 算符. 令 $\hat{W}=r^{k}$ 及 $\hat{W}=r^{k+1} \frac{d}{d r}$, 先后代入(11)式, 可得如下二式:

$$
\begin{aligned}
& k(k+1)\left\langle n l\left|r^{k-2}\right| n l\right\rangle+2 k\left\langle n l\left|r^{k-1} \frac{d}{d r}\right| n l\right\rangle=0, \\
& 2 z^{*}(2 k+1)\left\langle n l\left|r^{k-1}\right| n l\right\rangle-k(k-1)\left\langle n l\left|r^{k-1} \frac{d}{d r}\right| n l\right\rangle \\
& \quad+2 E_{n}(k+1)\left\langle n l\left|r^{k}\right| n l\right\rangle-2 l^{*}\left(l^{*}+1\right) k\left\langle n l\left|r^{k-2}\right| n l\right\rangle-0 .
\end{aligned}
$$

两式消去 $\left\langle n l\left|r^{k-1} \frac{d}{d r}\right| n l\right\rangle$, 可得

$$
\begin{aligned}
& 4(k+1) \frac{z^{* 2}}{n^{* 2}}\left\langle n l\left|r^{k}\right| n l\right\rangle=4 z^{*}(2 k+1)\left\langle n l\left|r^{k-1}\right| n l\right\rangle \\
& \quad+\left[k(k+1)(k-1)-4 k l^{*}\left(l^{*}+1\right)\right]\left\langle n l\left|r^{k-2}\right| n l\right\rangle .
\end{aligned}
$$

分别令上式中 $k=-2$ 和 $k=-1$, 可得以下二式:

$$
\begin{gathered}
{\left[8 l^{*}\left(l^{*}+1\right)-6\right]\left\langle n l\left|r^{-4}\right| n l\right\rangle=12 z^{*}\left\langle n l\left|r^{-3}\right| n l\right\rangle-4 \frac{z^{* 2}}{n^{* 2}}\left\langle n l\left|r^{-2}\right| n l\right\rangle,} \\
l^{*}\left(l^{*}+1\right)\left\langle n l\left|r^{-3}\right| n l\right\rangle=z^{*}\left\langle n l\left|r^{-2}\right| n l\right\rangle .
\end{gathered}
$$

由(10)式、(15)式和(16)式,可得

$$
\xi_{n l}=\frac{z^{* 2}}{2 c^{2}} \cdot \frac{\left[4 l^{*}\left(l^{*}+1\right)-3-12 B\right] n^{* 2}+4 B l^{*}\left(l^{*}+1\right)}{\left[4 l^{*}\left(l^{*}+1\right)-3\right] l^{*}\left(l^{*}+1\right) n^{* 2}} \cdot\left\langle n l\left|r^{-2}\right| n l\right\rangle .
$$

由于 (14) 式中 $k<-1$ 和 $k \geqslant-1$ 之间的关系是间断的, 从中得不到 $\left\langle n l\left|r^{-2}\right| n l\right\rangle$ 与 $\left\langle n l\left|r^{-1}\right| n l\right\rangle$ 之间的关系. 所以再利用正负幕平均值之间的关系, 令 (5) 式中 $T-0$, 则有 


$$
\left\langle n l\left|r^{-2}\right| n l\right\rangle-\left(\frac{2 z^{*}}{n^{*}}\right) \cdot \frac{1}{2 l^{*}+1}\left\langle n l\left|r^{-1}\right| n l\right\rangle .
$$

再令(14)式中 $k=0$, 可得

$$
\left\langle n l\left|r^{-1}\right| n l\right\rangle=\frac{z^{*}}{n^{* 2}}
$$

由(17)式、(18)式和(19)式, 可得多电子原子旋-轨耦合系数的计算式为

$$
\xi_{n l}=\frac{z^{* 1}}{c^{2} n^{* 5}} \cdot \frac{\left[4 l^{*}\left(l^{*}+1\right)-3-12 B\right] n^{* 2}+4 B l^{*}\left(l^{*}+1\right)}{\left[4 l^{*}\left(l^{*}+1\right)-3\right] l^{*}\left(l^{*}+1\right)\left(2 l^{*}+1\right)}, l^{*}>\frac{1}{2} .
$$

该公式十分简单, 在计算具体原子或离子时, 只需事先确定好参数, 就可以很容易地求出任意 激发态的旋-轨耦合系数.

当体系处于氢原子或类氢离子情形时, 由于不存在电子与电子之间的相互作用, 所以, 量 子修正因子 $d$ 、屏蔽常数 $\sigma$ 和相对增长因子 $g$, 均为 0 . 因而, $B-0, z^{*}-z, n^{*}-n, l^{*}=$ $l$, 于是(20)式可化为

$$
\xi_{n l}-\frac{z^{4}}{c^{2} n^{3}} \cdot \frac{1}{(2 l+1)(l+1) l},
$$

即类氢离子的旋- 轨耦合系数的计算式, 它与文献 [5]中的公式一致.

应用举例: 以下使用 $(20)$ 式计算 B III、CIV、N V 和 O VI 等电子体系 $n p$ 的旋-轨耦合 能. 为了将上述元素共用一套参数, 我们将 (2)式中 $z^{*}$ 改写如下:

\begin{tabular}{|c|c|c|c|c|c|c|c|c|}
\hline \multirow[b]{2}{*}{$n$} & \multicolumn{2}{|c|}{ B III } & \multicolumn{2}{|c|}{$\mathrm{c} ! \mathrm{V}$} & \multicolumn{2}{|c|}{$N \mathrm{~V}$} & \multicolumn{2}{|c|}{ o vI } \\
\hline & 本工作 & 文献 [ 6] & 本工作 & 文献 [ 6] & 本工作 & 文献 [ 6] & 本工作 & 文献 [ 6 ] \\
\hline 2 & 34.732 & 34.1 & 107.264 & 107.1 & 258.254 & 258.4 & 530.555 & 532.5 \\
\hline 3 & 10.262 & 10.2 & 31.692 & 31.5 & 76.303 & 74.2 & 156.757 & 156.6 \\
\hline 4 & 4.320 & 4.3 & 13.341 & 13.3 & 32.119 & 32.0 & 65.986 & 63.9 \\
\hline 5 & 2.208 & 2.2 & 6.820 & 6.7 & 16.419 & 16.0 & 33.732 & 33.0 \\
\hline 6 & 1.276 & & 3.942 & & 9.491 & & 19.498 & \\
\hline 7 & 0.803 & & 2.480 & & 5.972 & & 12.268 & \\
\hline 8 & 0.538 & & 1.660 & & 3.998 & & 8.213 & \\
\hline 9 & 0.377 & & 1.166 & & 2.806 & & 5.765 & \\
\hline 10 & 0.275 & & 0.849 & & 2.045 & & 4.201 & \\
\hline
\end{tabular}

$$
z^{*}-\left(z_{i}-\sigma_{n p}\right)+g_{n p} \Delta z,
$$

表 1 B III,C IV、N V、O VI 等电子体系 $2 p-10 p$ 的旋-轨耦合能 $\left(\mathrm{cm}^{-1}\right)$

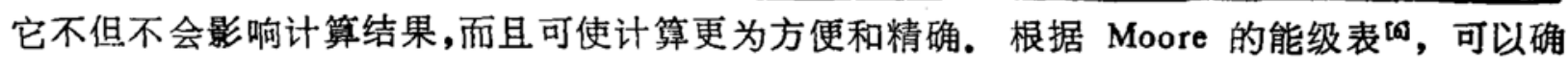
定参数为 $\sigma_{n p}-1.96, d_{n p}--0.016$ 和 $g_{n p}--0.01$, 并指定为首元素为 B III, 其核电荷数 为 $z_{0}, \Delta z$ 为其它元素的核电荷数 $z_{i}$ 与 $z_{0}$ 之差, 即 $\Delta z-z_{i}-z_{0}$. 计算结果列于表 1.

\section{参考文献}

[1; 郑能武,科学通报, 30( 1985),23: 1801 .

[2!郑能武,科学通报, 31(1986), 17: 1316 .

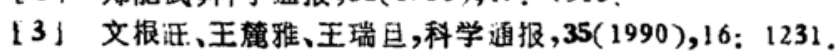

[4] Bockasten, K.. Phys. Rev.. A9(1974), 3: 1087-1089.

[5]徐光宪、黎乐民、王德民,量子化学—基本原理和从头计算法(中删), 科学出版社, 北京, 1985,659.

[6] Moore, C. E., Atomic Energy Levels, NSRDS-NBS35 1(1971), 19:58. 